\title{
Long noncoding RNA ENSRNOG00000037522 is involved in the podocyte epithelial-mesenchymal transition in diabetic rats
}

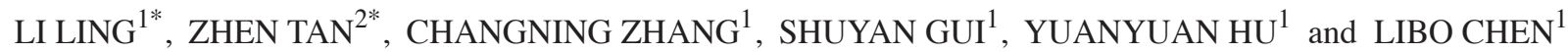 \\ ${ }^{1}$ Department of Endocrinology, Guangdong Medical College Affiliated Shenzhen Nanshan Hospital, Shenzhen, \\ Guangdong 518052; ${ }^{2}$ Department of Neurosurgery, Shenzhen Children's Hospital, Shenzhen, \\ Guangdong 518026, P.R. China
}

Received September 4, 2017; Accepted January 19, 2018

DOI: $10.3892 /$ ijmm.2018.3457

\begin{abstract}
Diabetic nephropathy (DN) is one of the most common complications associated with type I and II diabetes mellitus. Long noncoding RNAs (lncRNAs) have been implicated in various physiological and pathological processes, and recent evidence has demonstrated that they are involved in the process of the epithelial-mesenchymal transition (EMT). In the present study, the potential functions of lncRNA ENSRNOG00000037522 during the EMT process in DN were investigated. The results identified that the level of the lncRNA ENSRNOG00000037522 was significantly increased in kidney tissues collected from rats with streptozocin (STZ)-induced DN accompanied by impairment of the glomerular podocytes. It was further demonstrated that the silencing of lncRNA ENSRNOG00000037522 by small interfering RNA transfection partially restored the podocyte function. In addition, knockdown of lncRNA ENSRNOG00000037522 repaired the damage to the podocytes via regulating vimentin, podocalyxin-like 1 and nephrin expression. In conclusion, the current results demonstrated that IncRNA ENSRNOG00000037522 serves a pivotal role in the podocyte EMT in DN.
\end{abstract}

\section{Introduction}

The incidence of diabetes mellitus (DM) has markedly increased over the past few decades due to unhealthy diet, lack of exercise and deteriorating environmental conditions $(1,2)$. Among the various microvascular complications of the

Correspondence to: Dr Libo Chen, Department of Endocrinology, Guangdong Medical College Affiliated Shenzhen Nanshan Hospital, 89 Taoyuan Road, Nanshan, Shenzhen, Guangdong 518052, P.R. China

E-mail: chenlibo_1979@126.com

*Contributed equally

Key words: diabetic nephropathy, long noncoding RNA, ENSRNOG00000037522, podocytes, epithelial-mesenchymal transition disease, diabetic nephropathy (DN) is frequently observed and is the leading cause of end-stage renal disease (3). Podocytes are a key component of the kidney filtration barrier, and podocyte injury has been indicated to serve an important role in the pathogenesis of DN, according to a number of studies (4-8). Podocytes are terminally differentiated visceral epithelial cells that are located outside the glomerular capillaries and form the final filtration barrier to protein loss (9-11). In DN, podocyte injury leads to the disruption of the filtration barrier and to protein leakage. In addition, podocyte depletion has been indicated as an important early pathologic marker of DN $(7,12,13)$. Accumulating evidence also suggested that the epithelial-mesenchymal transition (EMT) is a possible cause of podocyte depletion in DN. During EMT, epithelial cells are transformed to mesenchymal cells in response to injurious stimuli $(10,13,14)$. Cells lose their original features when the pathological process of EMT occurs, consequently reducing cell-to-cell contact, damaging cell polarity and recapturing the characteristics of the mesenchymal markers, such as $\alpha$-smooth muscle actin ( $\alpha$-SMA) and vimentin (15).

Long noncoding RNAs (lncRNAs) are non-protein-coding transcripts with a length of $>200$ nucleotides that serve important roles in tumorigenesis $(16,17)$, RNA transcription $(18,19)$ and mRNA translation $(20,21)$. Furthermore, evidence has suggested that IncRNAs may be functionally important in DM progression through the modulation of renal responses to hyperglycemia and the progression of DN $(22,23)$. Several IncRNAs have been implicated in diabetic retinopathy, including MALAT1 (24), a potential biomarker for the prognosis and diagnosis of this disease. Wang et al (25) reported that a novel lncRNA, CYP4B1-PS1-001, was significantly downregulated in response to early $\mathrm{DN}$ in vivo and in vitro, and that overexpression of CYP4B1-PS1-001 inhibited the proliferation and fibrosis of mesangial cells. In addition, the lncRNAs H19 and HULC are known to be upregulated by oxidative stress, as well as to regulate cholangiocarcinoma migration and invasion (26). EMT is a potential pathway leading to podocyte depletion and proteinuria in diabetic kidney disease (27). However, the association of lncRNAs with the EMT in DN remains unclear.

In order to understand the functions of lncRNAs during the EMT process in streptozocin (STZ)-induced DN, IncRNA microarrays were initially used in the present study to identify 
the lncRNAs with differential expression between normal and DN rats. The results indicated that ENSRNOG00000037522 was the most upregulated lncRNA between normal and DN rats, and that the knockdown of ENSRNOG00000037522 affected the progression of EMT in DN. The current study provides novel insight into the mechanism linking lncRNA function with DN, and lncRNA ENSRNOG00000037522 may serve as a novel target for the development of DN.

\section{Materials and methods}

Rat model of diabetes. A total of 24 male Sprague Dawley rats (age, 8 weeks; weight, 180-200 g) were purchased from Forevergen Biotechnology Co., Ltd. (Guangzhou, China; http://www.forevergen.cn/). All animal studies were conducted under the review and approval of the Ethics Committee of Shenzhen Nanshan Hospital (Shenzhen, China). Rats were randomly allocated into three groups, including the control group, and two diabetes groups with rats examined at 1 or 6 weeks after STZ injection ( $n=8$ per group). Each group was subjected to controlled conditions of $20-22^{\circ} \mathrm{C}$, relative humidity of $50-55 \%$, and a 12-h light/dark cycle. After $24 \mathrm{~h}$ of fasting, diabetes in the two groups was induced by a single intraperitoneal injection of STZ $(50 \mathrm{mg} / \mathrm{kg}$ ) (Sigma-Aldrich, Merck KGaA, Darmstadt, Germany) that was dissolved in $5 \mathrm{mmol} / 1$ citrate buffer $(\mathrm{pH}=4.5)$ within $30 \mathrm{~min}$, while rats in the control group received intraperitoneal injection of the same dose of citrate buffer alone within 30 min (28). Rats were fed with Purina Rat Chow (Ralston Purina Company, Barcelona, Spain) throughout the experimental period. All rats had free access to food and water. Venous blood was collected at 24,48 or $72 \mathrm{~h}$ after STZ injection in order to assess whether the diabetes model had been successfully established (blood glucose level of $\geq 16.7 \mathrm{mmol} / \mathrm{l}$ ). Rats with STZ-induced diabetes were treated with $2 \mathrm{U} / \mathrm{kg}$ protamine zinc insulin at $24 \mathrm{~h}$ after STZ injection to avoid high glucose-induced mortality. At the end of 1 and 6 weeks after STZ or citrate buffer injection, all rats were euthanized with an intraperitoneal overdose of pentobarbital $(200 \mathrm{mg} / \mathrm{kg})$, and the kidneys were rapidly harvested and stored at $-80^{\circ} \mathrm{C}$ for use in subsequent experiments.

Cell culture. A total of $8 \times 10^{7}$ inactivated dynabeads were suspended in Hank's balanced salt solution (HBSS; Gibco; Thermo Fisher Scientific, Inc., Waltham, MA, USA). Next, the minced kidney specimens were digested with $1 \mathrm{mg} / \mathrm{ml}$ collagenase $\mathrm{A}$ at $37^{\circ} \mathrm{C}$ for $30 \mathrm{~min}$ and gently pressed through a $100-\mu \mathrm{m}$ cell strainer using a flattened pestle. Then the kidney lysate was mixed into HBSS and dynabeads. The mixture containing kidney lysate and dynabeads was centrifuged at $200 \mathrm{x} \mathrm{g}$ for $5 \mathrm{~min}$ at $4^{\circ} \mathrm{C}$, and the precipitates were resuspended in $5 \mathrm{ml} \mathrm{HBSS}$. Subsequent to washing three times with HBSS, the isolated glomeruli containing dynabeads were gathered by a magnetic particle concentrator and cultured in Dulbecco's modified Eagle medium (DMEM)/F12 (Gibco; Thermo Fisher Scientific, Inc.) at $37^{\circ} \mathrm{C}$ and $5 \% \mathrm{CO}_{2}$. After 7 days of culture, the glomeruli were digested in HBSS containing $1 \mathrm{mg} / \mathrm{ml}$ collagenase A and $0.2 \mathrm{mg} / \mathrm{ml}$ deoxyribonuclease I at $37^{\circ} \mathrm{C}$ for $60 \mathrm{~min}$, followed by centrifugation for $5 \mathrm{~min}$ at $1,500 \mathrm{x} \mathrm{g}$. The podocytes were then resuspended in DMEM/F12 medium and counted with a blood cell counter.
Finally, the morphology of the podocytes was examined at day 3, 5 and 7 with a scanning electron microscope (Nikon Corp., Tokyo, Japan) at x100 magnification. In addition, the podocytes were treated with high glucose $(30 \mathrm{mmol} / \mathrm{l})$ at day 7 for $2 \mathrm{~h}$ at room temperature.

Immunohistochemical analysis. All kidney tissues were fixed overnight in formalin solution, dehydrated in ethanol, embedded in paraffin and then sectioned at $5 \mathrm{~mm}$. The slides were blocked with $5 \%$ normal goat serum (Gibco, Thermo Fisher Scientific, Inc.) for $15 \mathrm{~min}$ at room temperature and incubated overnight at $4^{\circ} \mathrm{C}$ with primary antibodies, as follows: Anti- $\alpha$-SMA (bs-0189R; 1:200; Bioss Biotechnology Co., Ltd., Beijing, China), anti-vimentin (ab137321; 1:100; Abcam, Cambridge, MA, USA), anti-nephrin (ab216341; 1:100; Abcam), and anti-podocalyxin-like 1 (PODXL1; ab197769; 1:100; Abcam). Subsequent to washing with PBS, the slides were incubated with the rabbit anti-rat horseradish peroxidase (HRP)-conjugated IgG secondary antibody (ab6734; 1:500; Abcam) at room temperature for $20 \mathrm{~min}$. A DAB kit (Sigma-Aldrich; Merck KGaA, Darmstadt, Germany) was used to detect immunohistochemical reactions. The slides were then examined under a phase contrast light microscope (Eclipse E600; Nikon Corp.) at x200 magnification.

ELISA. Blood and urine samples were obtained at 1 and 6 weeks from the rats in each group. Blood was obtained from a tail vein. Serum was immediately separated by centrifugation at $6,000 \mathrm{x} \mathrm{g}$ for $20 \mathrm{~min}$ and stored at $-80^{\circ} \mathrm{C}$ until required for measurement. Urine samples were collected using metabolic cages. Kidney injury was assessed by measuring the levels of serum cystatin $C(\mathrm{Cys} C)$, serum $\beta_{2}$-microglobulin $\left(\beta_{2}-\mathrm{MG}\right)$, blood urea nitrogen (BUN) and urine microalbumin (UmAlb). ELISA kits were used to determine the concentration of serum CysC (In-Ra0699; Innova Biotech Co., Ltd., Beijing, China), serum $\beta_{2}$-MG (48-MICRT-E01; Alpco Diagnostics, Salem, NH, USA) and UmAlb (MBS9304276; MyBioSource, Inc., San Diego, CA, USA) with the enzyme immunoassay method according to the manufacturer's protocol. The content of the samples was then calculated with a spectrophotometer at $450 \mathrm{~nm}$. BUN concentration was measured using a Urea Assay kit (ab83362; Abcam) with a spectrophotometer at $570 \mathrm{~nm}$. These concentrations were quantified against a standard curve calibrated with known amounts of protein. Measurements were performed in triplicate.

Western blot analysis. To detect the glomerular proteins, western blot analysis was conducted. Briefly, cells were harvested and lysed in radioimmunoprecipitation assay buffer (Gibco, Thermo Fisher Scientific, Inc.) [containing $0.6 \mathrm{~g}$ Tris

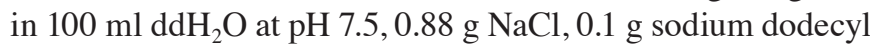
sulphate (SDS), $0.5 \mathrm{~g}$ sodium deoxycloate and $1.0 \mathrm{~g}$ NP-40 Tergitol] and a protease inhibitor mixture (Sigma-Aldrich; Merck KGaA). Next, the podocyte extracts were centrifuged at $4^{\circ} \mathrm{C}$ for $15 \mathrm{~min}$ at $1,000 \mathrm{x} \mathrm{g}$, and the supernatant was collected. The protein concentration was then determined with a bicinchoninic acid protein assay kit (Sangon Biotech Co., Ltd., Shanghai, China). Subsequently, $20 \mu \mathrm{g}$ protein was separated by $10 \%$ SDS-polyacrylamide gel electrophoresis and transferred to a polyvinylidene difluoride membrane (Bio-Rad 
Laboratories, Inc., Shanghai, China). After blocking in 1\% blocking reagent in Tris-buffered saline [10 mM Tris ( $\mathrm{pH} 7.5)$, $150 \mathrm{mM} \mathrm{NaCl}$ ] (Gibco, Thermo Fisher Scientific, Inc.) for $1 \mathrm{~h}$ at room temperature, the protein samples were then incubated overnight at $4^{\circ} \mathrm{C}$ with primary antibodies for anti- $\alpha$-SMA (no. 14968, 1:500; Cell Signaling Technology, Inc., Danvers, MA, USA), anti-vimentin (no. ab92547, 1:500; Abcam), anti-PODXL1 (no. SAB2500809, 1: 500; Sigma-Aldrich; Merck KGaA), anti-nephrin (no. ab136927, 1:500; Abcam) and GAPDH (ab9485, 1:500; Abcam). This was followed by incubation with a HRP-labeled goat anti-rabbit secondary antibody (1:1,000; Santa Cruz Biotechnology, Inc., Dallas, TX, USA) for $1 \mathrm{~h}$ at room temperature. Finally, the proteins were visualized with an enhanced chemiluminescence detection kit (Thermo Fisher Scientific, Inc.) and analyzed using ImageJ software, version 14.8 (National Institutes of Health, Bethesda, MD, USA).

Microarray IncRNA profiling. The lncRNA target sequences were merged from multiple databases, including RefSeq Build 37 (https://www.ncbi.nlm.nih.gov/refseq/), Ensembl Release 55 (http://www.ensembl.org), Unigene Build 176 (https://www. ncbi.nlm.nih.gov/unigene), GenBank (https://www.ncbi.nlm. nih.gov/gene) and RIKEN 3 (http://www.riken.jp). After 6 weeks, the podocytes were obtained from control and STZ-treated diabetic rats. Total RNA was extracted using TRIzol reagent (Takara Bio, Inc., Kusatsu, Japan), the concentration was measured using a NanoDrop spectrophotometer ND-1000 (Thermo Fisher Scientific, Inc.). Total RNA was converted into cDNA using a Takara reverse transcription kit (Takara Bio, Inc.), after which cRNA was synthesized from $\sim 2 \mu \mathrm{g}$ total RNA using an mMessage mMachine kit (Ambion, Thermo Fisher Scientific, Inc.) according to the manufacturer's standard protocols. Next, cRNA was hybridized to the microarray overnight, and microarray profiling was performed using the RiboArray ${ }^{\mathrm{TM}}$ Rat lncRNA Array (http://www.ribobio.com/sitecn/Service2.aspx?id=104; RiboBio Co., Ltd. (Guangzhou, China) following the manufacturer's protocol. The microarray after hybridization were washed with a Gene Expression Wash Buffer kit (Agilent Technologies, Inc., Santa Clara, CA, USA), then scanned in a microarry scanner using Agilent scan control software version 8.1 (Agilent Technologies, Inc., Santa Clara, CA, USA), and data were extracted with Feature Extraction software (version 10.7; Agilent Technologies, Inc.). For advanced data analysis, all biological replicates were pooled and calculated to identify differentially expressed lncRNAs, based on the threshold of a fold change of $\geq 2$ and $P \leq 0.05$. A hierarchical clustering heat map of the lncRNA expression profile was produced using fold changes in lncRNA expression. In addition, in order to determine the potential roles of differentially expressed lncRNAs, Gene Ontology (GO) and Kyoto Encyclopedia of Genes and Genomes (KEGG) pathway analyses were both applied. Enrichment for GO terms was analyzed using GOEAST software 20 (http://omicslab.genetics.ac.cn/GOEAST/). Enrichment for KEGG pathways using the KOBAS 3.0 Annotation System (http://kobas.cbi.pku.edu.cn).

LncRNA ENSRNOG00000037522 was identified and then its role was investigated using small interfering (si)RNA transfection experiments. The primary podocytes were
Table I. Sequences of the primers used in quantitative polymerase chain reaction.
Name
Sequences $\left(5^{\prime}-3^{\prime}\right)$

ENSROG00000011753 F: GGTGTTACGCTGGTCTTCCA R: ACCTGTCCTCATCCAAACCC

ENSROG00000050935 F: CGCAGAAGGTAACCTCCGTA R: TTTTTGGACCCGTCGCTTCT

LOC498759 F: ATGGAAGTGTGCAAGTCCT CA R: TCAGGCAAACGAGCACTC $\mathrm{AC}$

ENSROG00000031644 F: CTTAAGGATGCCTGGGCGAA R: CACATCAGCCACTGGGTAGT

ENSROG00000048366 F: CAATGGACGGCCATCGTTCT R: AGGAAAGACCTTGTGAGGC $\mathrm{AC}$

ENSROG00000039080 F: CGGCAAGGGAAGGTCAAT $\mathrm{CA}$

R:GAAGGCCTCAGTGGGTTTGT

ENSROG00000037522 F: CTCCCCAATCACCCAGCAT $\mathrm{TC}$

R: AGCGGGCTTTCTCTTTAATA TGCT

GAPDH

F: GCAAGAGAGAGGCCCTCAG

R: TGTGAGGGAGATGCTCAG

TG

F, forward; R, reverse.

Table II. Blood glucose ( $\mathrm{mmol} / \mathrm{l})$ in different groups of Sprague Dawley rats $(n=12)$.

\begin{tabular}{lccc}
\hline Group & $24 \mathrm{~h}$ & $48 \mathrm{~h}$ & $72 \mathrm{~h}$ \\
\hline Control & $6.77 \pm 0.25$ & $6.77 \pm 0.09$ & $6.73 \pm 0.33$ \\
STZ-injected & $23.53 \pm 1.06^{\mathrm{a}}$ & $25.03 \pm 1.46^{\mathrm{a}}$ & $25.30 \pm 1.22^{\mathrm{a}}$
\end{tabular}

${ }^{\mathrm{a}} \mathrm{P}<0.05$ vs. the control group. STZ, streptozocin.

transfected with siRNA targeting ENSRNOG00000037522. The negative control siRNA and two siRNAs against lncRNA ENSRNOG00000037522, namely siRNA1 and siRNA2, were designed by RiboBio Co., Ltd.. The sequences were as follows: siRNA1 sense, 5'-CCCAGCAUUCCAGCAUCUU-3', and antisense, 5'-AAGAUGCUGGAAUGCUGGG-3'; siRNA2 sense, 5'-GCAUUCCAGCAUCUUACCU-3', and antisense, 5'-AGG UAAGAUGCUGGAAUGC-3'. Briefly, podocytes were plated into 24-well plates at a density of $1 \times 10^{6}$ cells per well and grown overnight at room temperature, followed by addition of DMEM/F12 medium (Gibco; Thermo Fisher Scientific, Inc.) the following day. The podocytes were then transfected with control or ENSRNOG00000037522 siRNAs for $24 \mathrm{~h}$, using 

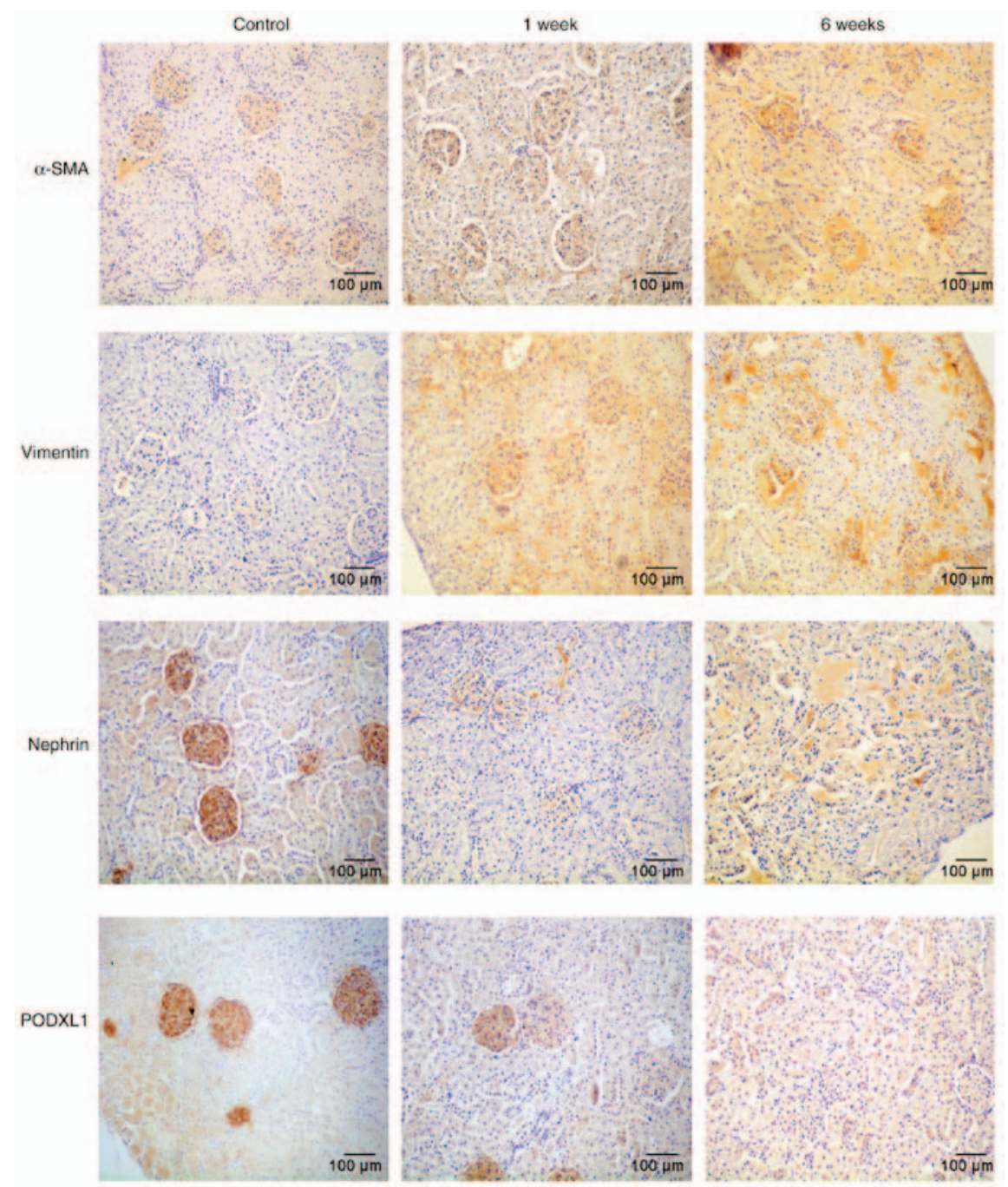

Figure 1. Protein expression of $\alpha$-SMA, vimentin, PODXL1 and nephrin, as examined by immunohistochemical assay. The $\alpha$-SMA and vimentin expression levels were almost absent in the control group, but were highly expressed in diabetic kidneys at 1 and 6 weeks. However, PODXL1 and nephrin were intensely expressed in the control rats, but markedly downregulated in the kidneys following STZ injection. All experiments were performed in triplicate, and representative immunohistochemistry images are shown. $\alpha$-SMA, $\alpha$-smooth muscle actin; PODXL1, podocalyxin-like 1; STZ, streptozocin.
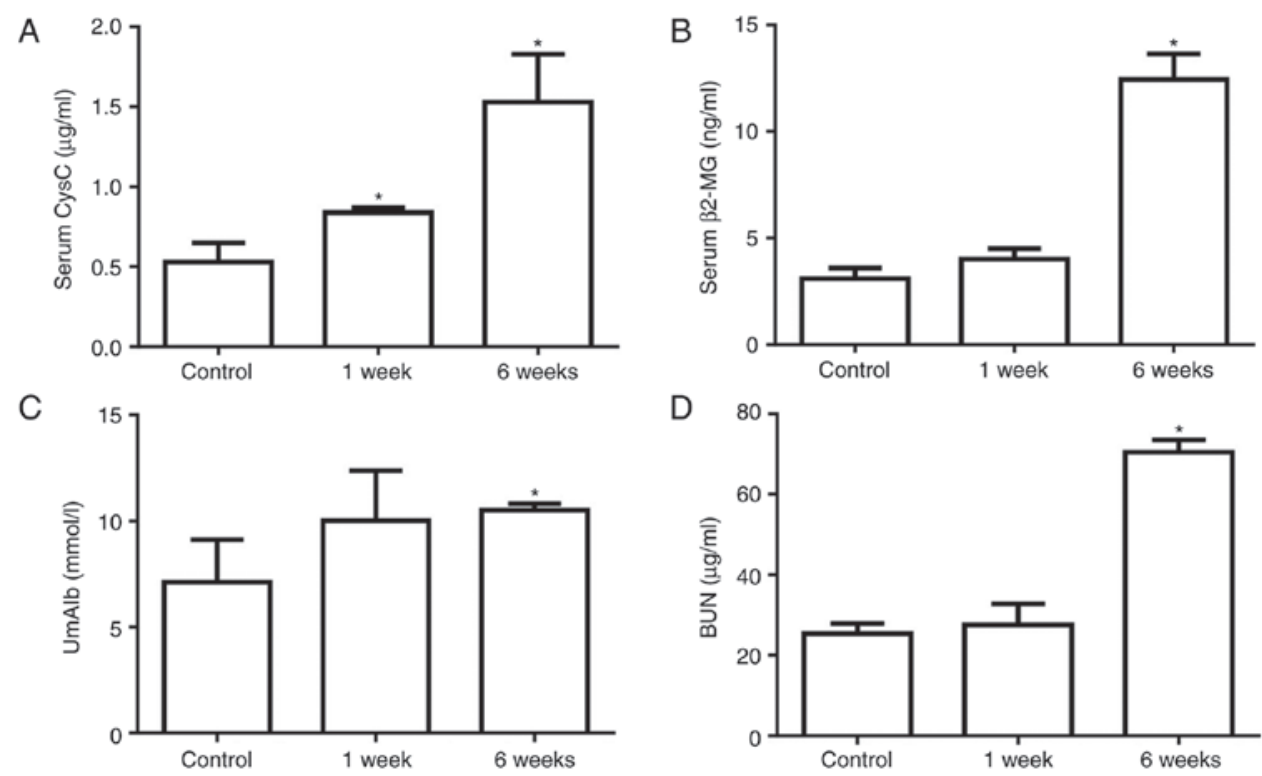

Figure 2. (A) Serum CysC, (B) serum $\beta 3-\mathrm{MG}$, (C) UmAlb and (D) BUN concentrations were determined by ELISA. These factors were increased in diabetic rats compared with the control rats. All measurements were performed in triplicate, and the results are presented as the mean \pm standard deviation. " $\mathrm{P}<0.05 \mathrm{vs}$. control group. CysC, cystatin C; $\beta_{2}$-MG, $\beta_{2}$-microglobulin; UmAlb, urine microalbumin; BUN, blood urea nitrogen. 

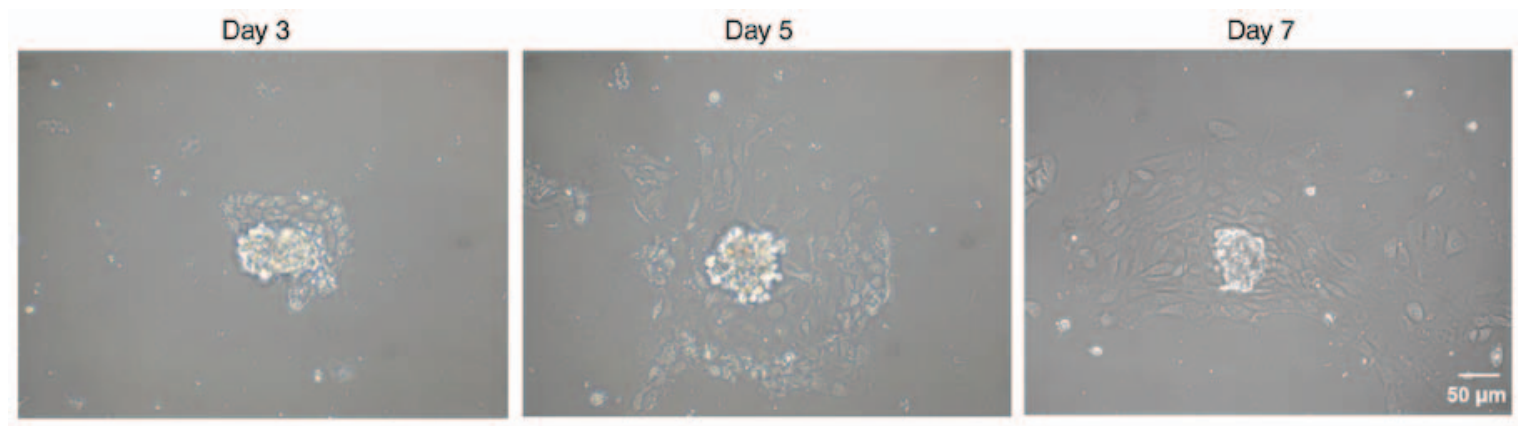

Figure 3. Morphology of podocytes on days 3 and 5 after incubation, and on day 7 after subculture was observed under a microscope (magnification, x100). Scale bar, $50 \mu \mathrm{m}$. All experiments were performed in triplicate, and representative images are shown.

Lipofectamine 2000 (Invitrogen; Thermo Fisher Scientific, Inc.) according to the manufacturer's protocol. Subsequent to transfection, the podocytes were grown for $72 \mathrm{~h}$ at $37^{\circ} \mathrm{C}$ and washed three times with PBS. The cells were then recovered in DMEM/F12 medium (Gibco; Thermo Fisher Scientific, Inc.) for $2 \mathrm{~h}$ and collected for use in subsequent experiments. The silencing efficiency was confirmed by reverse transcription-quantitative polymerase chain reaction (RT-qPCR), and all reactions were performed in triplicate.

$R N A$ extraction and $R T-q P C R$ analysis. Total RNA was isolated from podocytes using TRIzol reagent (Invitrogen; Thermo Fisher Scientific, Inc.), and the quantity and purity of the RNA were determined by optical density measurements at an A260/A280 ratio of $\geq 1.8$ using a NanoDrop 2000 spectrophotometer (Thermo Fisher Scientific, Inc.). Next, cDNA was synthesized using HiScript Q RT SuperMix for qPCR (Vazyme Biotech Co., Ltd., Nanjing, China) according to the manufacturer's protocol. The SYBR Green-based qPCR reaction system consisted of a final volume of $20 \mu \mathrm{l}$, containing $2 \mu \mathrm{l}$ cDNA, $10 \mu$ l SYBR-Green Mix (Takara Bio, Inc., Otsu, Japan), $4 \mu \mathrm{l}$ primer mix and $4 \mu \mathrm{ldd} \mathrm{d}_{2} \mathrm{O}$. The qPCR reaction was performed at $95^{\circ} \mathrm{C}$ for $5 \mathrm{~min}$, followed by 30 cycles of $95^{\circ} \mathrm{C}$ for $30 \mathrm{sec}$ and $50^{\circ} \mathrm{C}$ for $30 \mathrm{sec}$. Primers targeting lncRNAs were designed and synthetized by Thermo Fisher Scientific, Inc., and the sequences of the primers used are listed in Table I. The lncRNA expression levels were quantified using the $2^{-\Delta \Delta C q}$ method (29), with GAPDH serving as the endogenous reference gene.

Statistical analysis. Each experiment was performed three times. Student's t-test or one-way analysis of variance was used to analyze the data using SPSS version 19.0 software (IBM Corp., Armonk, NY, USA). All results were summarized and are presented as the mean \pm standard deviation. $\mathrm{P}<0.05$ was considered to indicate a difference that was statistically significant.

\section{Results}

Diabetes promotes EMT in rat kidneys. Rat blood glucose was monitored at 24, 48 and $72 \mathrm{~h}$ after STZ injection, and the results are shown in Table II. STZ-injected rats with blood glucose levels of $\geq 16.7 \mathrm{mmol} / 1$ were considered to be diabetic. Analysis at $24 \mathrm{~h}$ after STZ injection indicated that the blood glucose level had increased significantly as compared with the control rats, and continued to be elevated throughout the measurement period, which indicated that diabetes was established successfully in the STZ-injected rats.

In order to investigate the effects of diabetes on the EMT and the expression of PODXL1 in kidney tissues, an immunohistochemical assay was used to determine the expression levels of the mesenchymal phenotypic markers $\alpha$-SMA and vimentin, the epithelial marker nephrin and the podocyte-specific marker PODXL1. Yellow or brown staining was observed in cells that were positive for $\alpha$-SMA, vimentin, nephrin or PODXL1, while the nuclei were stained blue. The immunohistochemistry results demonstrated that expression of $\alpha$-SMA and vimentin was almost absent in the control tissues, whereas these markers were highly expressed in diabetic kidneys. By contrast, PODXL1 and nephrin were intensely expressed in the control rats, whereas they were markedly downregulated in the kidney tissues following STZ injection (Fig. 1).

Diabetes promotes kidney injury. At 1 or 6 weeks after STZ injection, the kidney function and injury were assessed by measuring the serum CysC, serum $\beta_{2}$-MG, UmAlb and BUN levels. The serum CysC level was $0.53 \pm 0.21 \mu \mathrm{g} / \mathrm{ml}$ in the control group, which was significantly increased to $0.76 \pm 0.04 \mu \mathrm{g} / \mathrm{ml}$ at 1 week after STZ injection and continued to increase after 6 weeks in the diabetic group (Fig. 2A). The serum $\beta_{2}-\mathrm{MG}, \mathrm{UmAlb}$ and BUN levels were not significantly different between the control and 1 week diabetic groups, but were significantly increased in diabetic rats at 6 weeks after STZ injection (Fig. 2B-D). These data indicated that kidney injury was induced by STZ stimulation and was exacerbated in diabetic rats after 6 weeks.

Next, glomeruli were isolated from the kidney tissues to obtain podocytes. Podocyte growth was detected by cell morphological observation at day 3,5 and 7 after culture. Subsequent to STZ injection, podocytes demonstrated foot process effacement, vacuolar degeneration and detachment (Fig. 3). In order to further confirm the process of EMT in diabetic rats, western blot analysis was performed to detect the expression levels of $\alpha$-SMA, vimentin, nephrin and PODXL1. The results revealed that the expression levels of nephrin and POXLD1 were significantly decreased in the podocytes of diabetic rats when compared with those in the control rats $(\mathrm{P}<0.05$; Fig. 4). By contrast, the expression levels of $\alpha$-SMA and vimentin were markedly upregulated in 

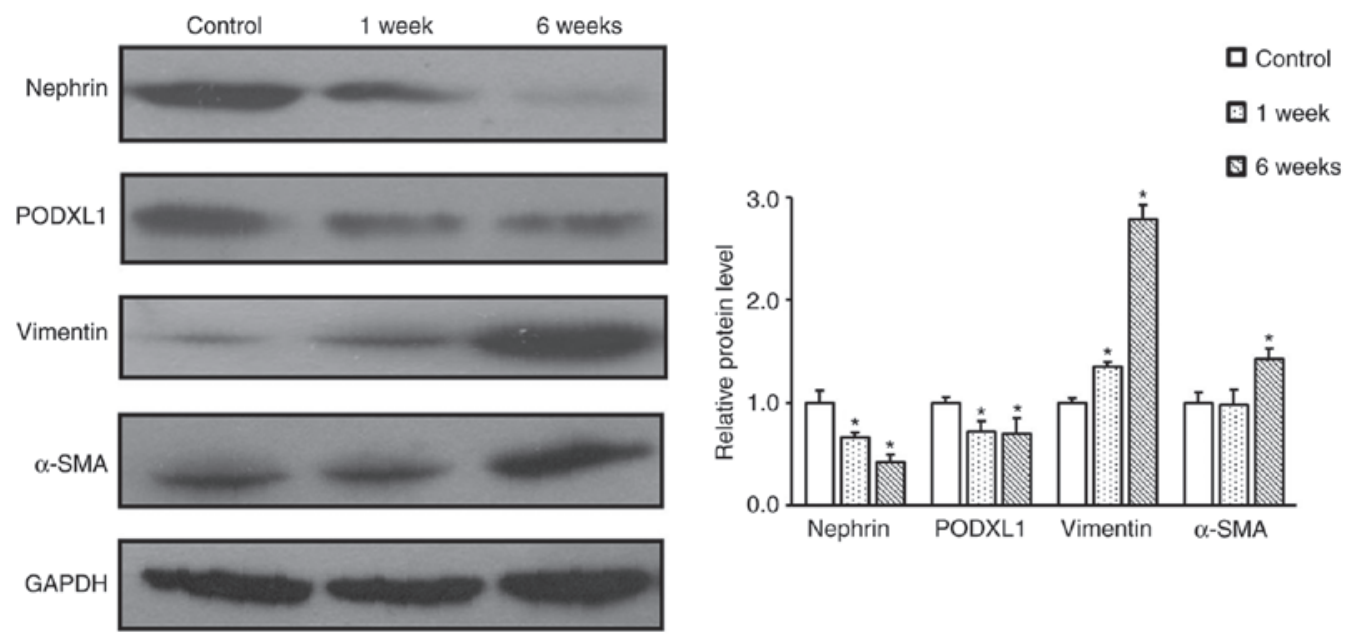

Figure 4. Nephrin, PODXL1, vimentin and $\alpha$-SMA protein expression levels in the podocytes of diabetic and control rats were examined with western blot analysis. All experiments were performed in triplicate, and the results are presented as the mean \pm standard deviation. " $\mathrm{P}<0.05$ vs. control group. PODXL1, podocalyxin-like $1 ; \alpha$-SMA, $\alpha$-smooth muscle actin.

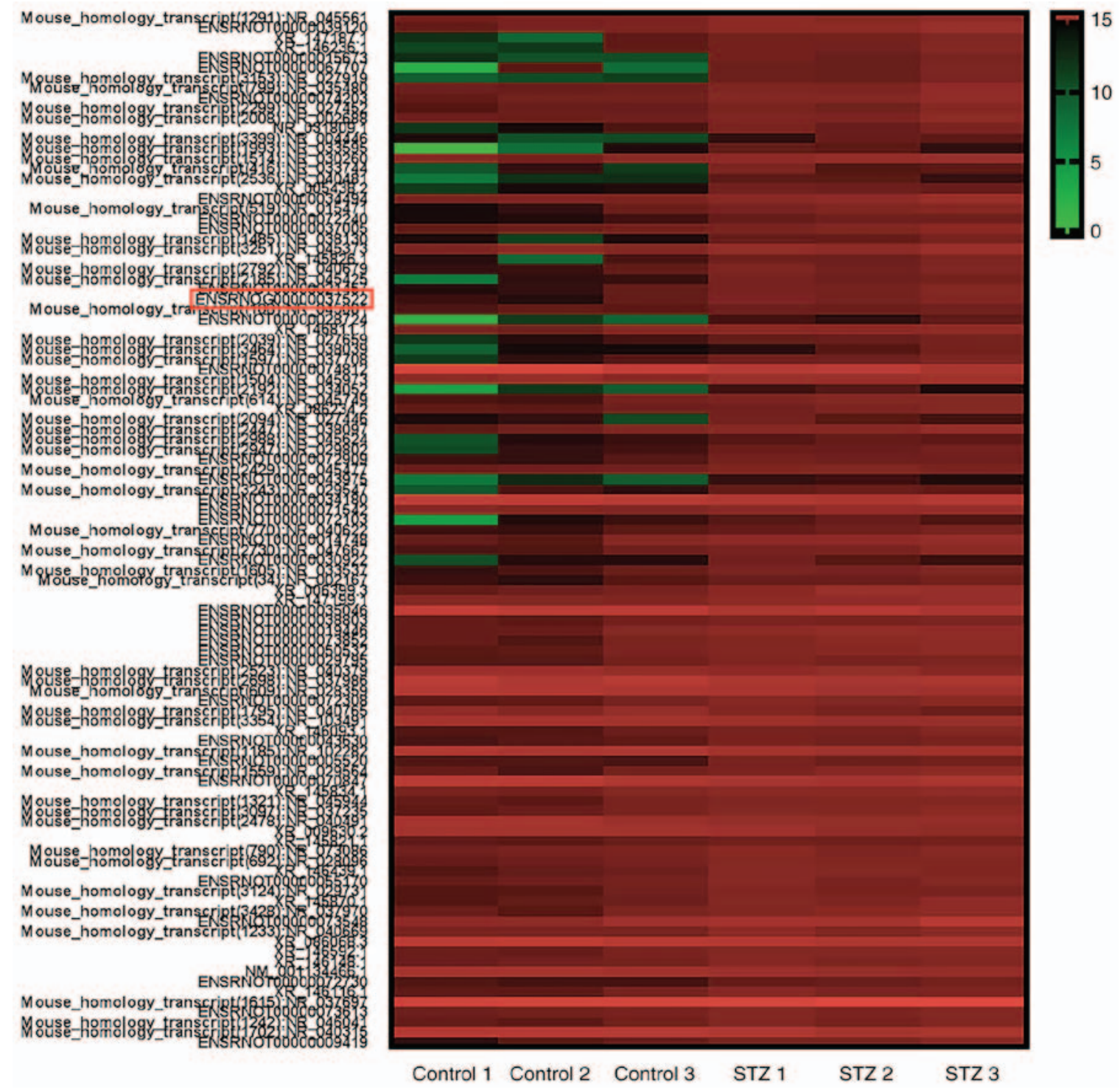

Figure 5. Hierarchical clustering heat map revealing the distinct lncRNA expression profiles in the podocytes of the control and STZ-treated diabetic rats. After 6 weeks, the podocytes were obtained from control and STZ-treated diabetic rats and hierarchical clustering analysis was performed. The relative lncRNA expression is depicted according to the color scale. The red column indicates upregulation and the green column indicates downregulation. IncRNA, long noncoding RNA; STZ, streptozocin. Control 1, 2 and 3 indicated three representative podocytes samples from control rats; STZ 1, 2 and 3 indicated three representative podocytes samples from STZ-treated diabetic rats. 


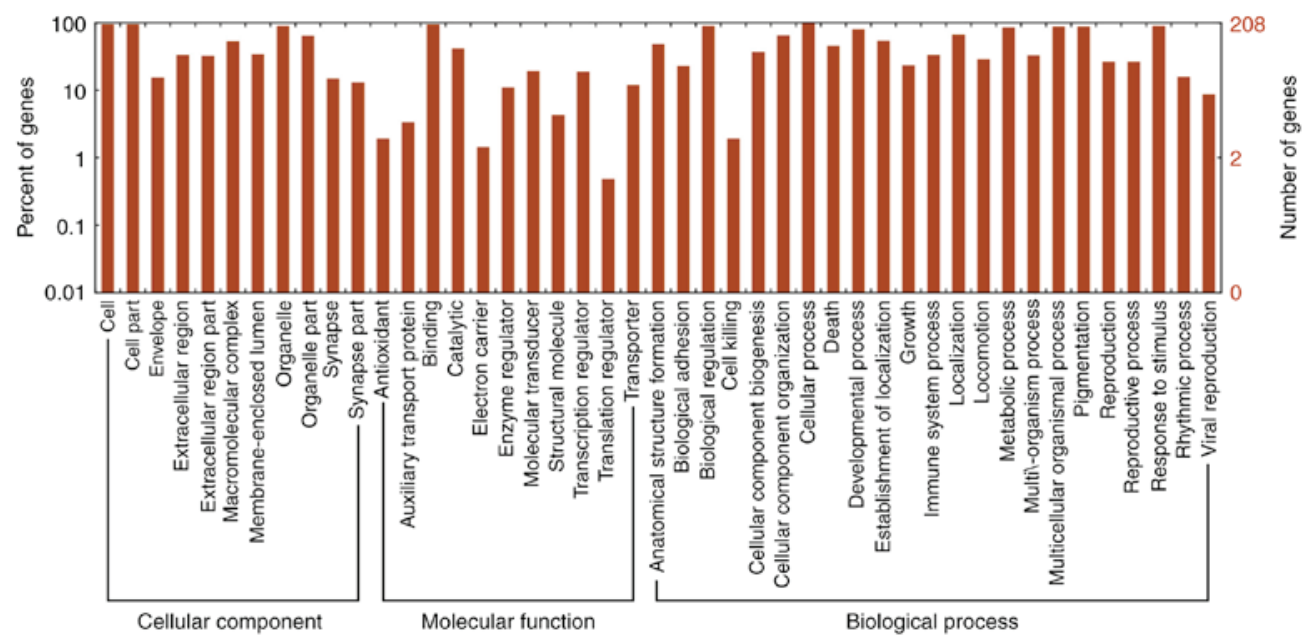

Figure 6. GO analysis of differentially expressed lncRNAs between the control and STZ-treated diabetic rats. Three domains were covered, including cellular component, molecular function and biological process. The GO analysis indicated that differentially expressed lncRNAs were mainly involved in the cell, cell part and cellular process. GO, Gene Ontology; lncRNA, long noncoding RNA; STZ, streptozocin.

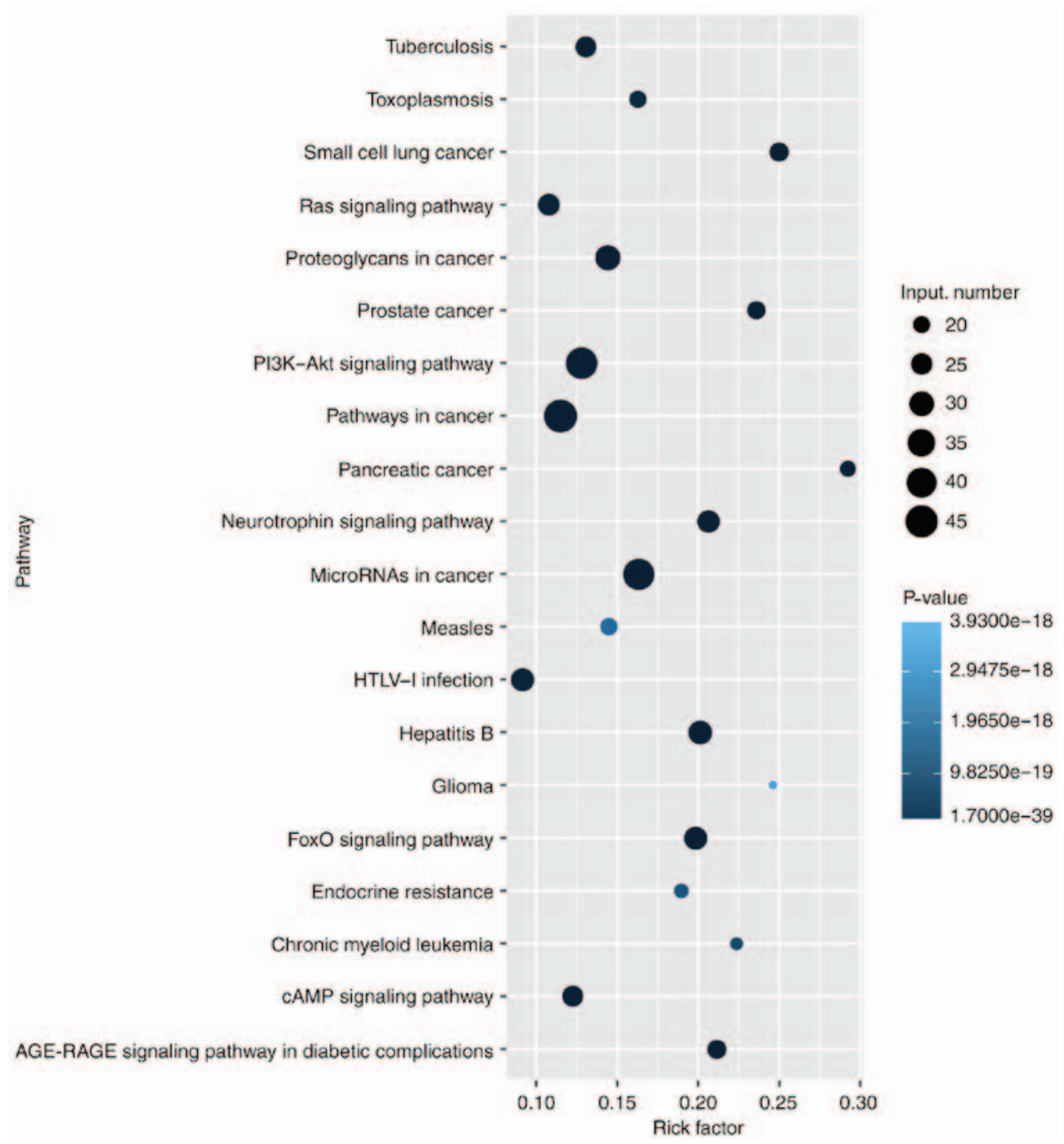

Figure 7. Kyoto Encyclopedia of Genes and Genomes pathway analysis of differentially expressed lncRNAs between the control and STZ-treated diabetic rats. The three most enriched pathways were 'pathways in cancer', 'PI3K-Akt signaling pathway' and 'microRNAs in cancer'. IncRNA, long noncoding RNA; STZ, streptozocin.

the podocytes of diabetic rats, as compared with those of the control rats (Fig. 4). These findings indicate that STZ-induced podocyte-specific depletion of nephrin and PODXL1 leads to the aggravation of kidney injury in diabetic rats. 
A

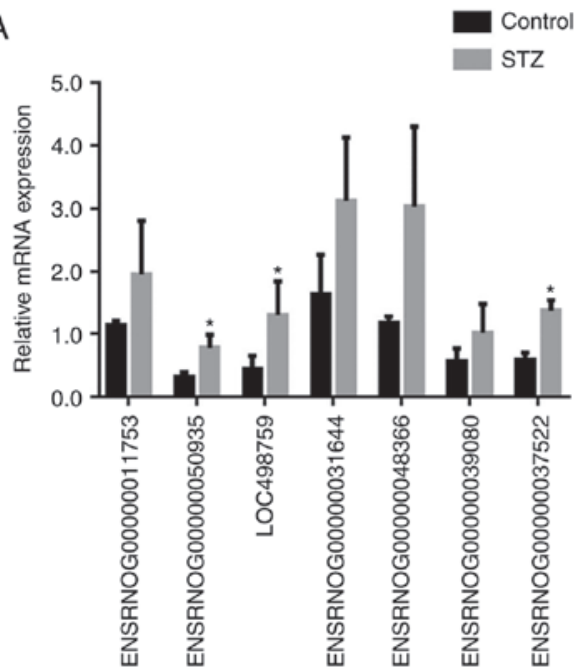

B

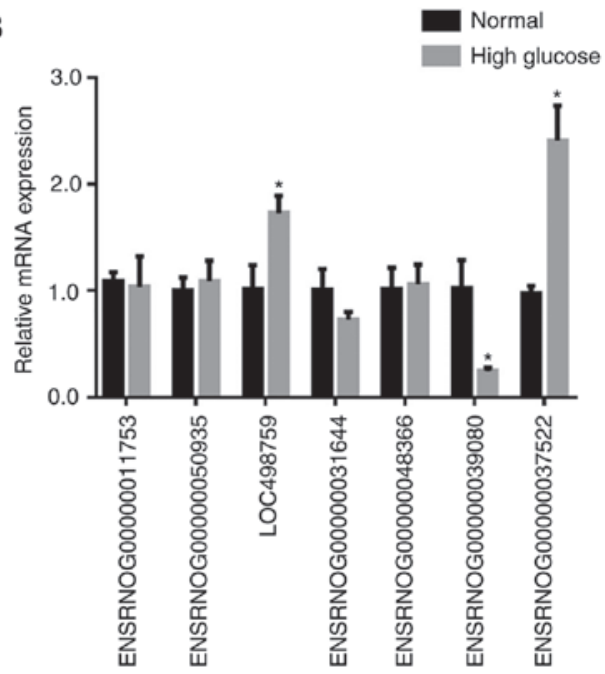

Figure 8. (A) RT-qPCR was used to validate the 7 selected lncRNAs transcripts. "P<0.05 vs. control group. (B) Subsequent to treatment of podocytes with high glucose, RT-qPCR validation was performed for the 7 selected lncRNA transcripts. IncRNA ENSRNOG00000037522 was the most upregulated lncRNA in podocytes from the high glucose treatment group compared with the normal group. ${ }^{*} \mathrm{P}<0.05$ vs. normal cells. All experiments were performed in triplicate, and the results are presented as the mean \pm standard deviation. IncRNA, long noncoding RNA; RT-qPCR, reverse transcription-quantitative polymerase chain reaction.

Differential expression of IncRNA in diabetic podocytes. To explore the potential function of lncRNAs during DN, a microarray analysis of the podocytes obtained from STZ-induced diabetic and control rats at 6 weeks was conducted. A total of 88 upregulated and 15 downregulated lncRNAs were identified between the two groups (data not shown). Heat map hierarchical clustering revealed distinct IncRNA expression profiles between the two groups (Fig. 5). Furthermore, the GO categories comprised three structured networks, including biological processes, the cellular component and the molecular function (Fig. 6). The GO analysis also indicated that differentially expressed lncRNAs were mainly involved in the cell, cell part, binding and cellular process (Fig. 6). Subsequently, KEGG pathway analysis was performed to ascertain the functions of the differentially expressed lncRNAs. The results suggested that 'pathways in cancer' were significantly enriched among these lncRNAs, and the three most enriched pathways included 'pathways in cancer', the 'PI3K-Akt signaling pathway' and 'microRNAs in cancer' (Fig. 7). Several of these pathways were linked to the EMT, such as the 'PI3K-Akt signaling pathway'. In order to verify the expression of the dysregulated lncRNAs, seven most upregulated lncRNAs from differentially expressed lncRNAs were selected for further investigation. The RT-qPCR results revealed variations in lncRNA expression in the podocytes of the STZ group, compared with that of the control group podocytes. As shown in Fig. 8A, there was a significant difference in the expression of the lncRNA ENSRNOG00000037522, ENSRNOG00000050935 and LOC498759 between the two groups.

High glucose induces IncRNA expression indiabeticpodocytes. To confirm the expression of the 7 selected lncRNAs, high glucose $(30 \mathrm{mmol} / \mathrm{l})$ was used to stimulate podocytes. Subsequently, RT-qPCR was performed to detect the mRNA expression levels of the 7 lncRNAs in normal podocytes and high glucose-stimulated podocytes. The results were in accordance with the microarray data. As shown in Fig. 8B, the IncRNA ENSRNOG00000037522 was the most upregulated lncRNA in podocytes from the high glucose group compared with those of the normal group. Consequently, these observations indicated that high glucose induced the expression of IncRNA ENSRNOG00000037522 in diabetic podocytes, and this IncRNA was selected for further investigation.

Knockdown of lncRNA ENSRNOG00000037522 inhibits podocyte EMT. Considering that the lncRNA ENSRNOG00000037522 levelwasincreased afterhigh-glucose stimulation, transfection with two siRNAs was applied to knockdown lncRNA ENSRNOG00000037522. The knockdown efficiency was confirmed by RT-qPCR, which revealed a marked reduction in lncRNA ENSRNOG00000037522 levels subsequent to siRNA1 and siRNA2 transfection (Fig. 9A). The western blot analysis results also demonstrated that the expression levels of PODXL1 and nephrin were significantly increased, whereas the $\alpha$-SMA and vimentin expression levels were decreased following siRNA transfection (Fig. 9B and C). These aforementioned observations indicate that the knockdown of lncRNA ENSRNOG00000037522 influenced the EMT in podocytes by downregulating vimentin and upregulating nephrin.

\section{Discussion}

DN is a common kidney disease caused by hyperglycemia that can lead to angiopathy of the glomerular capillaries (30). Glomerular endothelial cells, the glomerular basement membrane and podocytes constitute the filtration barrier. Podocytes form the final barrier to protein loss and podocyte dysfunction allows penetration into the other slit structures $(9,31)$. Numerous studies have suggested that podocyte depletion is responsible for the onset of proteinuria, and the EMT has been proposed to be a possible engagement for podocyte depletion in DN $(7,13,14,32,33)$. 
A
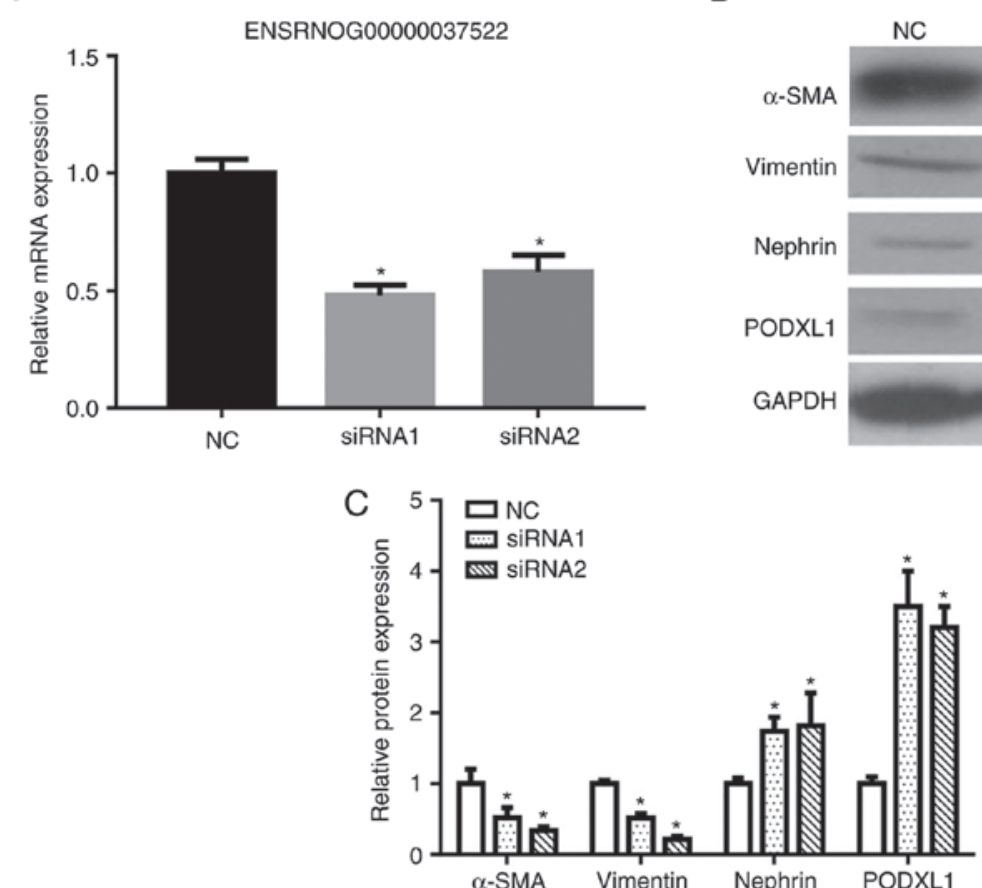

B

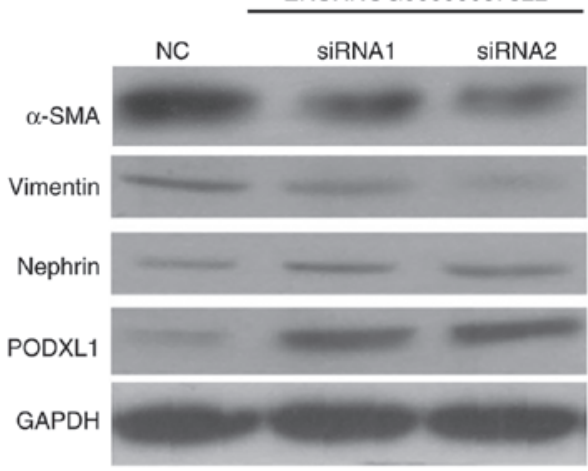

Figure 9. (A) The knockdown efficiency of the lncRNA ENSRNOG00000037522 was examined with reverse transcription-quantitative polymerase chain reaction, and a significant abrogation of lncRNA ENSRNOG00000037522 was observed following transfection with siRNA1 or siRNA2. (B) Western blot analysis was conducted to determine the $\alpha$-SMA, vimentin, nephrin and POXLD1 expression levels in lncRNA ENSRNOG00000037522 knockdown and control podocytes. (C) Protein levels were quantified by ImageJ software and are presented in the histogram. Podocytes transfected with NC siRNA served as the controls. All experiments were performed in triplicate, and the results are presented as the mean \pm standard deviation. ${ }^{*} \mathrm{P}<0.05 \mathrm{vs.} \mathrm{NC} \mathrm{group.} \mathrm{lncRNA}$, long noncoding RNA; NC, negative control; siRNA, small interfering RNA.

In the present study, it was demonstrated that podocytes were damaged in the kidneys of rats with STZ-induced diabetes, while the downregulation of PODXL1 and nephrin expression levels, as well as the upregulation of $\alpha$-SMA and vimentin expression levels were observed. Nephrin is the first protein to be identified at the glomerular podocyte slit diaphragm and its expression has been described to be transiently increased in the first 8 weeks subsequent to the establishment of a diabetes animal model. The downregulation of nephrin expression was identified in the kidneys of type 1 and type 2 diabetes patients (34). In addition, nephrin is a podocyte-specific protein; thus, a reduction in its expression may reflect podocyte loss in diabetic rats, and this loss can lead to a range of renal diseases, including DN (35). Podocalyxin (PODX) is expressed by kidney podocytes, vascular endothelia and a subset of neurons (36). Aberrant expression of PODX contributes to podocyte-associated renal diseases (37). PODXL1 is an anti-adhesive transmembrane protein that has been reported to be expressed on the foot processes of podocytes in the kidney glomerulus, as well as on the endothelium at certain sites (36). The results of the present study revealed that PODXL1 expression was decreased in diabetic rats following STZ injection, which is consistent with the observations of a previous study (38).

The levels of several serum and urine response factors, including serum CysC, UmAlb, serum $\beta_{2}-\mathrm{MG}$ and BUN, were also examined in the present study and were observed to be increased in diabetic rats. Serum CysC and $\beta_{2}-\mathrm{MG}$ have been reported to be increased in kidney injury $(39,40)$. Notably, serum $\mathrm{CysC}$ is superior to serum creatinine for the diagnosis of early diabetic nephropathy, and an elevated serum CysC concentration is a strong predictor of DN (41). Therefore, in the present study serum CysC was found to be increased at 1 and 6 weeks after STZ injection, whereas UmAlb, serum $\beta_{2}-\mathrm{MG}$ and BUN levels were significantly increased only at 6 weeks after STZ injection. It is, thus, speculated that the downregulation of PODXL1 and nephrin expression in podocytes leads to elevated levels of urine response factors, thereby aggravating kidney injury.

Numerous studies have been published recently on the functional roles of lncRNAs in DN. For instance, Zhou et al (42) identified that the IncRNA MIAT served an essential role in high glucose-induced renal tubular epithelial injury in a rat model of diabetes. Wang et al (25) also demonstrated that the CYP4B1-PS1-001 lncRNA inhibited the proliferation and fibrosis of mouse mesangial cells during the early stages of DN. In addition, Beltrami et al (43) reported that LipcRNAp21 lncRNA was associated with diabetes-induced vascular complications. Hu et al (44) observed that MALAT1 lncRNA served a pivotal role in DN and high glucose-induced podocyte damage. According to the aforementioned findings, a microarray profiling analysis of the differential expression of lncRNAs in diabetic rats was conducted in the current study. It was determined that IncRNA ENSRNOG00000037522 was significantly upregulated in the STZ-induced DN group compared with the control group. It was further confirmed that STZ- or high glucose-induced podocyte EMT occurred via the upregulation 
of lncRNA ENSRNOG00000037522, and was accompanied by the downregulation of PODXL1 and nephrin expression and the upregulation of $\alpha$-SMA and vimentin expression. In addition, IncRNA ENSRNOG00000037522 knockdown repaired podocyte damage via the downregulation of $\alpha$-SMA and vimentin, and the upregulation of PODXL1 and nephrin expression levels.

EMT is an important biological process during the development of DN, and podocytes undergoing EMT lose the phenotypic characteristics of epithelial cells, exhibiting reduced nephrin and PODX expression, while they also express phenotypic markers of mesenchymal cells, including $\alpha$-SMA and vimentin (10). Nevertheless, the mechanisms that mediate podocyte EMT remain poorly understood. Given that $\alpha$-SMA and vimentin are markers of mesenchymal cells, their expression levels were observed to be increased in high glucose-induced EMT in podocytes in the current study, which was consistent with the findings of a previous study (27). These results indicate that $\alpha$-SMA and vimentin are essential for STZ-induced EMT, and it is speculated that STZ-induced EMT leads to the loss of functional proteins, which, in turn, damage the function and structure of podocytes. However, further research is required to investigate whether influencing the EMT is an efficient treatment strategy for DN.

In the current study, GO and KEGG pathway analyses were also used to identify the potential functions of the differentially expressed lncRNAs. Several terms in the GO results were involved in the EMT process, including the cellular process term. The KEGG pathway analysis also revealed that numerous pathways were associated with the EMT, including the 'PI3K-Akt signaling pathway'. It has been reported that the PI3K-Akt signaling pathway is essential in the process of EMT, and inhibition of this signaling pathway was able to reduce podocytes damage (45). These results indicated the general role of ENSRNOG00000037522 in STZ-induced EMT and podocyte damage.

In conclusion, the expression of the lncRNA ENSRNOG00000037522 was upregulated in the kidneys of rats with STZ-induced diabetes. Furthermore, the results indicated that the inhibitory effects of IncRNA ENSRNOG00000037522 on high glucose-induced podocyte EMT may provide further evidence for the prevention and treatment of DN by targeting lncRNA ENSRNOG00000037522.

\section{Acknowledgements}

The present study was supported by the Youth Science Fund Project of National Natural Science Fund of China (grant no. 81400818) and the Provincial Natural Science Foundation of Guangdong (grant no.2017A030313783).

\section{Competing interests}

The authors declare that they have no competing interests.

\section{References}

1. Forbes JM and Cooper ME: Mechanisms of diabetic complications. Physiol Rev 93: 137-188, 2013.

2. Guariguata L, Whiting DR, Hambleton I, Beagley J, Linnenkamp U and Shaw JE: Global estimates of diabetes prevalence for 2013 and projections for 2035. Diabetes Res Clin Pract 103: 137-149, 2014.
3. Collins AJ, Foley RN, Chavers B, Gilbertson D, Herzog C, Johansen K, Kasiske B, Kutner N, Liu J, St Peter W, et al: United States Renal Data System 2011 Annual Data Report: Atlas of chronic kidney disease \& end-stage renal disease in the United States. Am J Kidney Dis 59 (Suppl 1): A7, e1-420, 2012.

4. Eid AA, Ford BM, Block K, Kasinath BS, Gorin Y, Ghosh-Choudhury G, Barnes JL and Abboud HE: AMP-activated protein kinase (AMPK) negatively regulates Nox4-dependent activation of p53 and epithelial cell apoptosis in diabetes. J Biol Chem 285: 37503-37512, 2010.

5. Eid AA, Gorin Y, Fagg BM, Maalouf R, Barnes JL, Block K and Abboud HE: Mechanisms of podocyte injury in diabetes: Role of cytochrome P450 and NADPH oxidases. Diabetes 58: 1201-1211, 2009.

6. Kim DK, Nam BY, Li JJ, Park JT, Lee SH, Kim DH, Kim JY, Kang HY, Han SH, Yoo TH, et al: Translationally controlled tumour protein is associated with podocyte hypertrophy in a mouse model of type 1 diabetes. Diabetologia 55: 1205-1217, 2012.

7. Susztak K, Raff AC, Schiffer $M$ and Bottinger EP: Glucose-induced reactive oxygen species cause apoptosis of podocytes and podocyte depletion at the onset of diabetic nephropathy. Diabetes 55: 225-233, 2006.

8. Weil EJ, Lemley KV, Mason CC, Yee B, Jones LI, Blouch K, Lovato T, Richardson M, Myers BD and Nelson RG: Podocyte detachment and reduced glomerular capillary endothelial fenestration promote kidney disease in type 2 diabetic nephropathy. Kidney Int 82: 1010-1017, 2012.

9. Faul C, Asanuma K, Yanagida-Asanuma E, Kim K and Mundel P: Actin up: Regulation of podocyte structure and function by components of the actin cytoskeleton. Trends Cell Biol 17: 428-437, 2007.

10. Li Y, Kang YS, Dai C, Kiss LP, Wen X and Liu Y: Epithelial-to-mesenchymal transition is a potential pathway leading to podocyte dysfunction and proteinuria. Am J Pathol 172: 299-308, 2008.

11. Pavenstädt H, Kriz W and Kretzler M: Cell biology of the glomerular podocyte. Physiol Rev 83: 253-307, 2003.

12. Durvasula RV and Shankland SJ: Podocyte injury and targeting therapy: An update. Curr Opin Nephrol Hypertens 15: $1-7,2006$.

13. Kang YS, Li Y, Dai C, Kiss LP, Wu C and Liu Y: Inhibition of integrin-linked kinase blocks podocyte epithelial-mesenchymal transition and ameliorates proteinuria. Kidney Int 78: 363-373, 2010.

14. Yamaguchi Y, Iwano M, Suzuki D, Nakatani K, Kimura K, Harada K, Kubo A, Akai Y, Toyoda M, Kanauchi M, et al: Epithelial-mesenchymal transition as a potential explanation for podocyte depletion in diabetic nephropathy. Am J Kidney Dis 54: 653-664, 2009.

15. Dai H, Liu Q and Liu B: Research progress on mechanism of podocyte depletion in diabetic nephropathy. J Diabetes Res 2017: 1-10, 2017.

16. Boon RA, Jaé N, Holdt L and Dimmeler S: Long Noncoding RNAs: From clinical genetics to therapeutic targets? J Am Coll Cardiol 67: 1214-1226, 2016.

17. Qiu MT, Hu JW, Yin R and Xu L: Long noncoding RNA: An emerging paradigm of cancer research. Tumour Biol 34: 613-620, 2013.

18. Gibb EA, Warren RL, Wilson GW, Brown SD, Robertson GA, Morin GB and Holt RA: Activation of an endogenous retrovirus-associated long non-coding RNA in human adenocarcinoma. Genome Med 7: 22, 2015.

19. Zhang H, Chen Z, Wang X, Huang Z, He Z and Chen Y: Long non-coding RNA: A new player in cancer. J Hematol Oncol 6: 37, 2013.

20. Amit-Avraham I, Pozner G, Eshar S, Fastman Y, Kolevzon N, Yavin E and Dzikowski R: Antisense long noncoding RNAs regulate var gene activation in the malaria parasite Plasmodium falciparum. Proc Natl Acad Sci USA 112: E982-E991, 2015.

21. Hung CL, Wang LY, Yu YL, Chen HW, Srivastava S, Petrovics G and Kung HJ: A long noncoding RNA connects c-Myc to tumor metabolism. Proc Natl Acad Sci USA 111: 18697-18702, 2014.

22. Alvarez ML and Distefano JK: The role of non-coding RNAs in diabetic nephropathy: Potential applications as biomarkers for disease development and progression. Diabetes Res Clin Pract 99: 1-11, 2013.

23. Ding GL, Wang FF, Shu J, Tian S, Jiang Y, Zhang D, Wang N, Luo Q, Zhang Y, Jin F, et al: Transgenerational glucose intolerance with Igf2/H19 epigenetic alterations in mouse islet induced by intrauterine hyperglycemia. Diabetes 61: 1133-1142, 2012. 
24. Yan B, Tao ZF, Li XM, Zhang H, Yao J and Jiang Q: Aberrant expression of long noncoding RNAs in early diabetic retinopathy. Invest Ophthalmol Vis Sci 55: 941-951, 2014.

25. Wang M, Wang S, Yao D, Yan Q and Lu W: A novel long non-coding RNA CYP4B1-PS1-001 regulates proliferation and fibrosis in diabetic nephropathy. Mol Cell Endocrinol 426: 136-145, 2016.

26. Wang WT, Ye H, Wei PP, Han BW, He B, Chen ZH and Chen YQ: LncRNAs H19 and HULC, activated by oxidative stress, promote cell migration and invasion in cholangiocarcinoma through a ceRNA manner. J Hematol Oncol 9: 117, 2016.

27. Chen T, Zheng LY, Xiao W, Gui D, Wang X and Wang N: Emodin ameliorates high glucose induced-podocyte epitheliamesenchymal transition in-vitro and in-vivo. Cell Physiol Biochem 35: 1425-1436, 2015.

28. Roy S, Rahaman N, Ahmed F, Metya S and Sannigrahi S: Naringenin attenuates testicular damage, germ cell death and oxidative stress in streptozotocin induced diabetic rats: naringenin prevents diabetic rat testicular damage. J Applied Biomedicine 11: 195-208, 2013.

29. Livak KJ and Schmittgen TD: Analysis of relative gene expression data using real-time quantitative PCR and the $2^{-\Delta \Delta C_{\mathrm{T}}}$ method. Methods 25: 402-408, 2001.

30. Xu X, Luo P, Wang Y, Cui Y and Miao L: Nuclear factor (erythroid-derived 2)-like 2 (NFE2L2) is a novel therapeutic target for diabetic complications. J Int Med Res 41: 13-19, 2013.

31. Mundel P and Shankland SJ: Podocyte biology and response to injury. J Am Soc Nephrol 13: 3005-3015, 2002.

32. Wiggins RC: The spectrum of podocytopathies: A unifying view of glomerular diseases. Kidney Int 71: 1205-1214, 2007

33. Yu D, Petermann A, Kunter U, Rong S, Shankland SJ and Floege J: Urinary podocyte loss is a more specific marker of ongoing glomerular damage than proteinuria. J Am Soc Nephrol 16: $1733-1741,2005$

34. Cooper ME, Mundel $P$ and Boner G: Role of nephrin in renal disease including diabetic nephropathy. Semin Nephrol 22: 393-398, 2002

35. Benigni A, Gagliardini E, Tomasoni S, Abbate M, Ruggenenti P, Kalluri R and Remuzzi G: Selective impairment of gene expression and assembly of nephrin in human diabetic nephropathy. Kidney Int 65: 2193-2200, 2004.

36. Sassetti C, Tangemann K, Singer MS, Kershaw DB and Rosen SD: Identification of podocalyxin-like protein as a high endothelial venule ligand for L-selectin: Parallels to CD34. J Exp Med 187: 1965-1975, 1998.
37. Hara M, Yamagata K, Tomino Y, Saito A, Hirayama Y, Ogasawara S, Kurosawa H, Sekine S and Yan K: Urinary podocalyxin is an early marker for podocyte injury in patients with diabetes: Establishment of a highly sensitive ELISA to detect urinary podocalyxin. Diabetologia 55: 2913-2919, 2012.

38. Xing Y, Ye S, Chen Y, Hu W and Chen Y: Hydrochloride pioglitazone protects diabetic rats against podocyte injury through preserving glomerular podocalyxin expression. Arq Bras Endocrinol Metabol 58: 630-639, 2014.

39. Ataei N, Bazargani B, Ameli S, Madani A, Javadilarijani F, Moghtaderi M, Abbasi A, Shams S and Ataei F: Early detection of acute kidney injury by serum cystatin $\mathrm{C}$ in critically ill children. Pediatr Nephrol 29: 133-138, 2014.

40. Sharifiaghdas F, Kashi AH and Eshratkhah R: Evaluating percutaneous nephrolithotomy-induced kidney damage by measuring urinary concentrations of beta2-microglobulin. Urol J 8: 277-282, 2011.

41. Shimizu A, Horikoshi S, Rinnno H, Kobata M, Saito K and Tomino Y: Serum cystatin C may predict the early prognostic stages of patients with type 2 diabetic nephropathy. J Clin Lab Anal 17: 164-167, 2003.

42. Zhou L, Xu DY, Sha WG, Shen L, Lu GY and Yin X: Long non-coding MIAT mediates high glucose-induced renal tubular epithelial injury. Biochem Biophys Res Commun 468: 726-732, 2015.

43. Beltrami C, Angelini TG and Emanueli C: Noncoding RNAs in diabetes vascular complications. J Mol Cell Cardiol 89: 42-50, 2015.

44. Hu M, Wang R, Li X, Fan M, Lin J, Zhen J, Chen L and Lv Z: LncRNA MALAT1 is dysregulated in diabetic nephropathy and involved in high glucose-induced podocyte injury via its interplay with $\beta$-catenin. J Cell Mol Med 21: 2732-2747, 2017.

45. Li D, Lu Z, Xu Z, Ji J, Zheng Z, Lin S and Yan T: Spironolactone promotes autophagy via inhibiting PI3K/AKT/mTOR signalling pathway and reduce adhesive capacity damage in podocytes under mechanical stress. Biosci Rep 36: pii: 362016 , 2016.

This work is licensed under a Creative Commons

Attribution-NonCommercial-NoDerivatives 4.0 International (CC BY-NC-ND 4.0) License. 